\title{
Metal-insulator transition and superconductivity in boron-doped diamond
}

\author{
T. Klein, ${ }^{1,2}$ P. Achatz,,${ }^{1,3}$ J. Kacmarcik, ${ }^{1,4}$ C. Marcenat, ${ }^{3}$ F. Gustafsson, ${ }^{1}$ J. Marcus, ${ }^{1}$ E. Bustarret, ${ }^{1}$ J. Pernot, ${ }^{1}$ F. Omnes, ${ }^{1}$ \\ Bo E. Sernelius, ${ }^{5}$ C. Persson, ${ }^{6}$ A. Ferreira da Silva, ${ }^{7}$ and C. Cytermann ${ }^{8}$ \\ ${ }_{1}^{1}$ Institut Néel, CNRS, Boîte Postale 166, 38042 Grenoble Cedex 9, France \\ ${ }^{2}$ Institut Universitaire de France, Université Joseph Fourier, Boîte Postale 53, 38041 Grenoble Cedex 9, France \\ ${ }^{3}$ CEA-Grenoble, Département de Recherche Fondamentale sur la Matière Condensée, 38054 Grenoble Cedex 9, France \\ ${ }^{4}$ Center of Low Temperature Physics, IEP Slovakian Academy of Sciences, Watsonova 47, 04353 Kosice, Slovakia \\ ${ }^{5}$ Department of Physics, Chemistry and Biology, Linkoping University, 58183 Linkoping, Sweden \\ ${ }^{6}$ Department of Materials Science and Engineering, KTH, 10044 Stockholm, Sweden \\ ${ }^{7}$ Instituto de Fisica, Universidade Federal da Bahia, 40210340 Salvador, Bahia, Brazil \\ ${ }^{8}$ Solid State Institute, Technion, 32000 Haifa, Israel
}

(Received 3 November 2006; revised manuscript received 19 January 2007; published 17 April 2007)

\begin{abstract}
We report on a detailed analysis of the transport properties and superconducting critical temperatures of boron-doped diamond films grown along the $\{100\}$ direction. The system presents a metal-insulator transition (MIT) for a boron concentration $\left(n_{B}\right)$ on the order of $n_{c} \sim 4.5 \times 10^{20} \mathrm{~cm}^{-3}$, in excellent agreement with numerical calculations. The temperature dependence of the conductivity and Hall effect can be well described by variable range hopping for $n_{B}<n_{c}$ with a characteristic hopping temperature $T_{0}$ strongly reduced due to the proximity of the MIT. All metallic samples (i.e., for $n_{B}>n_{c}$ ) present a superconducting transition at low temperature. The zero-temperature conductivity $\sigma_{0}$ deduced from fits to the data above the critical temperature $\left(T_{c}\right)$ using a classical quantum interference formula scales as $\sigma_{0} \propto\left(n_{B} / n_{c}-1\right)^{\nu}$ with $\nu \sim 1$. Large $T_{c}$ values $(\geqslant 0.4 \mathrm{~K})$ have been obtained for boron concentration down to $n_{B} / n_{c} \sim 1.1$ and $T_{c}$ surprisingly mimics a $\left(n_{B} / n_{c}-1\right)^{1 / 2}$ law. Those high $T_{c}$ values can be explained by a slow decrease of the electron-phonon coupling parameter $\lambda$ and a corresponding drop of the Coulomb pseudopotential $\mu^{*}$ as $n_{B} \rightarrow n_{c}$.

DOI: 10.1103/PhysRevB.75.165313

PACS number(s): 71.30.+h, 71.20.Nr, 74.25.Fy
\end{abstract}

\section{INTRODUCTION}

The recent discovery of superconductivity in boron-doped diamond $^{1}$ in the vicinity of a metal-insulator transition (MIT) naturally raised the question of the correlation between these two electronic instabilities. However, in contrast to doped silicon or germanium, ${ }^{2}$ little work has been performed so far on the MIT in this system. An analysis of the MIT has been recently performed by Tshepe et al. ${ }^{3}$ in ion-implanted films, suggesting that the critical concentration for the MIT, $n_{c}$, might be on the order of $4 \times 10^{21} \mathrm{~cm}^{-3}$. The authors also obtained a surprisingly high value for the critical exponent $\nu$ $(\sim 1.7)$ in the scaling of the conductivity, suggesting that diamond belongs to a universality class different from that of other doped semiconductors.

However, we will show that in our single-crystal diamond epilayers, the zero-temperature conductivity, deduced from fits to the data above the superconducting critical temperature using a classical quantum interference formula, varies as $\left(n_{B} / n_{c}-1\right)$ for $n_{B}>n_{c}$, leading to a scaling exponent $\nu \sim 1$, i.e., close to the one previously observed in disordered metals ${ }^{4}$ and many semiconductors. ${ }^{5,6}$ We will also see that on the insulating side of the transition, the temperature dependence of the conductivity $(\sigma)$ and the Hall coefficient $\left(R_{H}\right)$ can be very well described by a variable range hopping mechanism ${ }^{7}$ with a characteristic hopping temperature which is strongly reduced due to the proximity of the MIT. Moreover, we obtained a critical value $n_{c}$ on the order of 4.5 $\times 10^{20} \mathrm{~cm}^{-3}$, in very good agreement with numerical calculations but 1 order of magnitude smaller than the one previously obtained by Tshepe et al. ${ }^{3}$ in their more disordered samples.
Theoretical calculations ${ }^{8-12}$ suggested that superconductivity arises from the coupling of phonons with holes in the top of the $\sigma$ bonding bands ${ }^{8-12}$ as observed in magnesium diboride. ${ }^{13}$ However, despite a very large electron-phonon coupling potential $(V)$, the three-dimensional (3D) nature of the $\mathrm{C}$ network in diamond ( $s p^{3}$ type) greatly reduces its density of states $\left(g_{F}\right)$ compared to the one of the quasi-twodimensional (quasi-2D) $\mathrm{MgB}_{2}$ compound ( $s p^{2}$ bonding of $\mathrm{B}$ atoms isostructural to graphite). The theoretical calculations thus lead to an electron-phonon coupling constant $\lambda=g_{F} V$ on the order of $0.4-0.5$ for $\sim 5 \%$ holes per carbon atoms ${ }^{8-12}$ much smaller than in $\mathrm{MgB}_{2}(\lambda \sim 1) . \lambda$ is even expected to further decrease as the MIT is approached and diamond thus appears to be an exotic system of fundamental interest for the study of the influence of low carrier concentration on superconducting properties of materials.

From the requirement that the superconducting gap vanishes for $T=T_{c}$, BCS theory predicts that the critical temperature $T_{c} \sim 0.85 \Theta_{D} \exp \left(-1 / g_{F} V\right)$, where $\Theta_{D}$ is the Debye temperature. This expression is only valid in the weak-coupling limit $\left(\lambda=g_{F} V \ll 1\right)$ and a semiempirical expression has been proposed by McMillan, solving numerically the Eliashberg equations: ${ }^{14}$

$$
T_{c} \sim \hbar \omega_{\text {log }} / 1.2 k_{B} \exp \left[-\frac{1.04(1+\lambda)}{\lambda-\mu^{*}(1+0.62 \lambda)}\right],
$$

where $\omega_{\log }$ is a logarithmic averaged phonon frequency (on the order of $1020 \mathrm{~cm}^{-1}$ in diamond) and $\mu^{*}$ is the Coulomb pseudopotential. We will show that $T_{c}$ remains abnormally large down to $n_{B} / n_{c} \sim 1.1\left(T_{c} \geqslant 0.4 \mathrm{~K}\right)$ due to a very good coupling and reduced Coulomb pseudopotential. We will see 
that the fast decrease of $\lambda$ expected in virtual-crystal calculations ${ }^{10-12}$ is unable to reproduce the experimental data, thus suggesting that local boron vibrational modes play a significant role in superconductivity in diamond.

\section{SAMPLE PREPARATION AND EXPERIMENTS}

A series of homoepitaxial boron-doped diamond films has been grown by microwave plasma-enhanced chemical-vapor deposition along the $\{100\}$ direction from a $\mathrm{H}_{2} / \mathrm{CH}_{4} / \mathrm{B}_{2} \mathrm{H}_{6}$ gas mixture. ${ }^{15}$ The very narrow $(10-20$ arc sec) $\{400\}$ diffraction peaks measured on these biaxially stressed epilayers ${ }^{16}$ confirm their high structural quality and chemical homogeneity. Transport measurements have then been performed using the standard four-probe configurations and the boron atomic concentrations $n_{B}$ were derived from secondary-ion-mass spectroscopy (SIMS) experiments as described in Ref. 15. Superconductivity has been observed in all metallic samples and the critical temperature $T_{c}$ has been deduced from transport [ $90 \%$ of the normal-state resistivity, labeled $R$, see Fig. 1(a)] and/or susceptibility (onset of diamagnetic screening, labeled $\chi$, see inset of Fig. 2 in Ref. 15 for a typical example) measurements. Those values have been reported in Table I. Both sets of measurements show a well defined superconducting transition and good agreement has been obtained from both criteria in sample 509 in which both measurements were performed. Moreover, a very similar $T_{c}$ value has also been deduced from the temperature dependence of the gap from tunneling spectroscopy measurements, ${ }^{17}$ clearly emphasizing the homogeneity of our films. No superconductivity could be observed down to $50 \mathrm{mK}$ for $n_{B} \leqslant 4 \times 10^{20} \mathrm{~cm}^{-3}$ (i.e., on the insulating side of the MIT) and, as discussed below, the temperature dependence of the resistivity then obeys an $\exp \left[-\left(T_{0} / T\right)^{1 / 4}\right]$ law, as expected for variable range hopping.

\section{INSULATING SIDE OF THE TRANSITION: VARIABLE RANGE HOPPING CONDUCTIVITY}

For $n_{B} \leqslant 4.5 \times 10^{20} \mathrm{~cm}^{-3}$, the conductivity decreases very sharply with decreasing temperature and several hopping mechanisms can then be considered, writing $\sigma$ $=\sigma_{0} \exp \left[-\left(T_{0} / T\right)^{m}\right]$. For a simple activated regime (i.e., for tunneling toward the nearest accessible site) $m=1$, but it has been shown by Mott $^{7}$ that it can be energetically favorable to hop over larger distances seeking for the most favorable site [variable range hopping, (VRH) regime, $m=1 / 4$ in three dimensions]. However, this model assumes that the density of states at the Fermi level $\left(g_{F}\right)$ is almost constant, but longrange unscreened Coulomb repulsion may strongly reduce $g_{F}$ (Coulomb gap), leading to $m=1 / 2$ [Efros-Shklovskii ${ }^{18}$ (ES) regime]. As shown in Fig. 1(b), we did observe that $\sigma(T)$ can be very well reproduced taking $m=1 / 4$ between $\sim 10$ and $300 \mathrm{~K}$ (with $T_{0} \sim 3700 \mathrm{~K}$ and $\sim 210 \mathrm{~K}$ for $n_{B}=2.4$ $\times 10^{20} \mathrm{~cm}^{-3}$ and $n_{B}=4 \times 10^{20} \mathrm{~cm}^{-3}$, respectively ${ }^{19}$ ).

It has been predicted by Gruenewald et al. ${ }^{20}$ in a percolation model that the Hall mobility $\left(\mu_{H}\right)$ should also follow a VRH law: $\ln \left(\mu_{H}\right) \propto-3 / 8\left(T_{0} / T\right)^{1 / 4}$, leading to a Hall coefficient $R_{H} \propto \exp \left[-\left(T_{0, H} / T\right)^{1 / 4}\right]$ with $\left(T_{0, H} / T_{0}\right) \sim(5 / 8)^{4} \sim 0.15$.
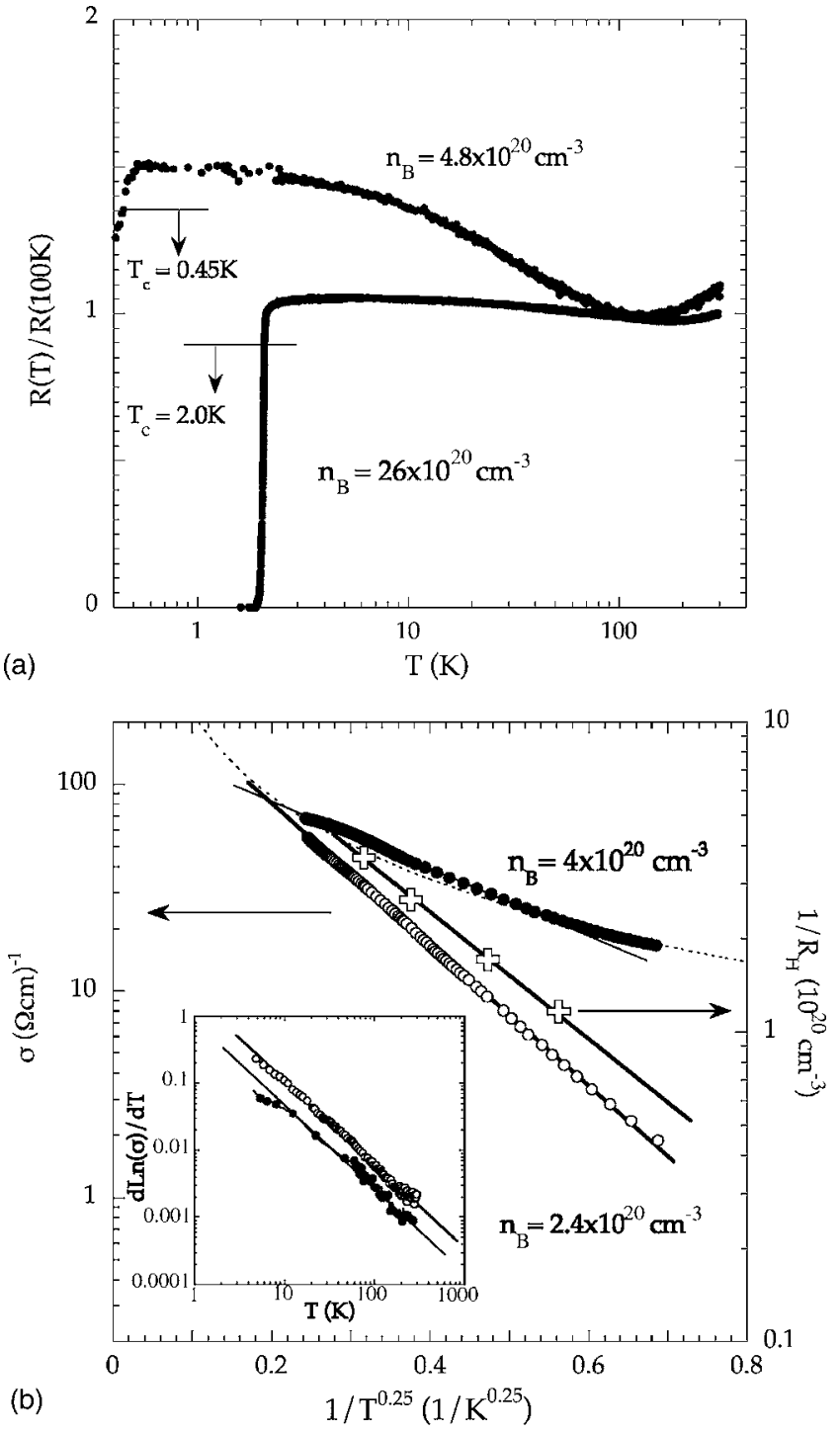

FIG. 1. (a) Temperature dependence of the electrical resistivity rescaled to its $T=100 \mathrm{~K}$ value of two samples clearly showing the onset of superconductivity at low temperature. (b) Semilogarithmic plot of the conductivity (left scale, circles) and inverse Hall coefficient (right scale, crosses) as a function of $1 / T^{0.25}$ for $n_{B}=2.4$ $\times 10^{20} \mathrm{~cm}^{-3}$ (open symbols) and $n_{B}=4 \times 10^{20} \mathrm{~cm}^{-3}$ (closed symbols, conductivity only). The solid lines are the expected behavior in the variable range hopping regime and the dashed line corresponds to $\sigma \propto T^{1 / 3}$. Inset: $\log -\log$ plot of $d \ln (\sigma) / d T$ as a function of $T$.

As shown in Fig. 1(b) (for $n_{B}=2.4 \times 10^{20} \mathrm{~cm}^{-3}$ ), the VRH law is indeed very well reproduced for both $\sigma$ and $1 / R_{H}$ with $T_{0} \sim 3700 \mathrm{~K}$ and $T_{0, H} \sim 500 \mathrm{~K}$, i.e., $T_{0, H} / T_{0} \sim 0.13$, in good agreement with the theoretical prediction. ${ }^{20,21}$

$T_{0}$ is related to the localization length $\xi_{\text {loc }}$ through $T_{0}$ $\sim\left(C_{M} / k_{B} g_{F} \xi_{l o c}^{3}\right)^{1 / 4}$, where $C_{M}$ is a numerical constant (even though percolation theories confirmed the initial proposition by Mott that $m=1 / 4$, there exists a considerable discrepancy on the $C_{M}$ value 22,23 ranging from $\sim 1$ to $\sim 28$ ). Far from the transition, $\xi_{l o c}$ is on the order of the Bohr radius $(\sim 3.5 \AA)$ and $g_{F} \approx n_{B} / w$ can be estimated assuming that the width $(w)$ of the impurity band caused by Coulomb interaction between 


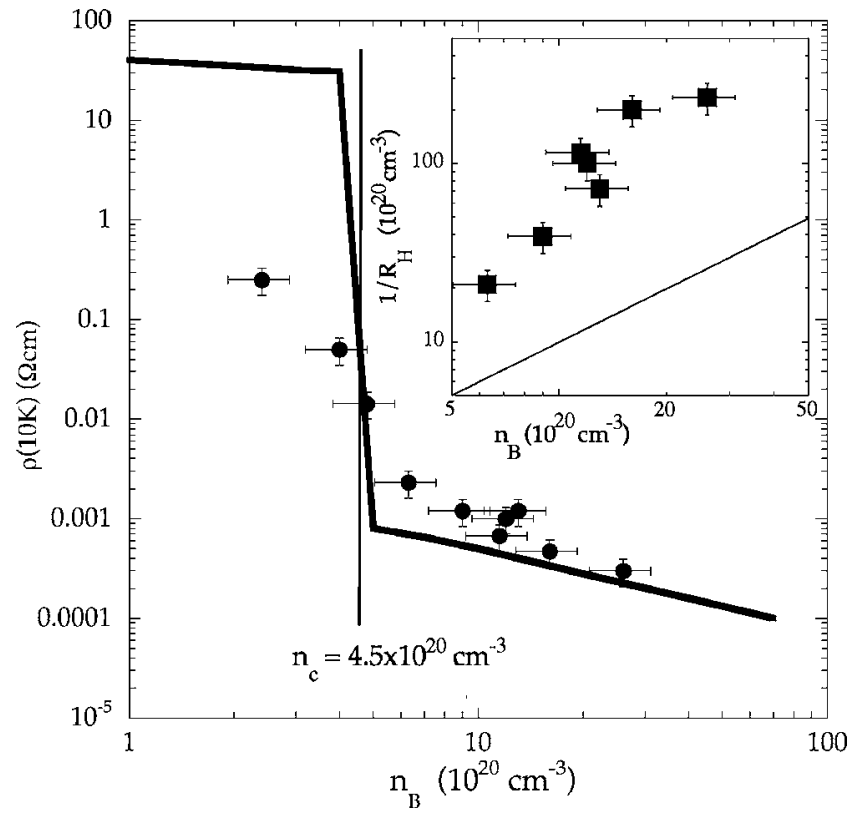

FIG. 2. Resistivity (at $T=10 \mathrm{~K}$ ) as a function of the boron content deduced from SIMS measurements $\left(n_{B}\right)$. The solid line corresponds to calculations in the generalized Drude model. A metalinsulator transition is predicted for $n_{B} \sim(4-5) \times 10^{20} \mathrm{~cm}^{-3}$, in good agreement with experimental data. Inset: Effective number of carriers $\left(n_{e f f} \propto 1 / R_{H}\right)$ deduced from Hall measurements as a function of $n_{B}$; the solid line would correspond to $n_{e f f}=n_{B}$.

nearest-neighbor boron impurities is $w \sim e^{2} / \kappa r_{B}$, where $r_{B}$ in the mean distance between impurities $\sim\left(3 / 4 \pi n_{B}\right)^{1 / 3}$ and $\kappa$ $=4 \pi \epsilon_{0} \epsilon_{r}$. One hence gets $T_{0}$ values on the order of $10^{6} \mathrm{~K}$, in good agreement with the value reported by Sato et al. ${ }^{24}$ but much larger than those that we obtained in our just-insulating samples.

However, close to the transition $\xi_{l o c}$ is expected to diverge, leading to very small $T_{0}$ values. The "distance" to the MIT can be quantified in terms of the boron concentration $\left(n_{B}\right)$ through the parameter $\left|n_{B} / n_{c}-1\right|$, where $n_{c}$ is a critical

TABLE I. Boron concentration deduced from SIMS measurements $\left(n_{B}\right.$ in $\left.10^{20} \mathrm{~cm}^{-3}\right)$, conductivity value extrapolated to $T$ $=0 \mathrm{~K}\left[\sigma_{0}\right.$ in $\left.(\mu \Omega \mathrm{cm})^{-1}\right]$, and superconducting critical temperature $\left(T_{c}\right.$ in $\left.\mathrm{K}\right)$ in a series of boron-doped diamond homoepitaxial films.

\begin{tabular}{lccc}
\hline \hline Sample & $n_{B}$ & $\sigma_{0}$ & $T_{c}$ \\
\hline 411 & 2.4 & $2(4 \mathrm{~K})$ & $\leqslant 50 \mathrm{mK}$ \\
662 & 4 & $17(4 \mathrm{~K})$ & $\leqslant 50 \mathrm{mK}$ \\
666 & 4.8 & 70 & $0.45(R)$ \\
400 & 6.3 & 430 & $0.55(R)$ \\
418 & 9 & 820 & $0.9(\chi)$ \\
420 & 11.5 & 1480 & $1.4(\chi)$ \\
412 & 12 & 1000 & $1.2(\chi)$ \\
419 & 13 & 870 & $1.2(\chi)$ \\
438 & 16 & 2130 & $1.3(\chi)$ \\
507 & 19 & Unknown thickness & $1.55(\chi)$ \\
509 & 26 & 3260 & $2.0(R+\chi)$ \\
\hline \hline
\end{tabular}

concentration. The critical regime can be described by two characteristic exponents, ${ }^{25} \nu$ and $\eta$. The former relates the correlation length $\left(\xi \equiv \xi_{l o c}\right)$ to the external parameter which drives the transition (here the concentration $n_{B}$ ) through $\xi$ $\propto 1 /\left|n_{B}-n_{C}\right|^{\nu}$ and the latter relates the energy scale to the length scale $\left(E \propto 1 / L^{\eta}\right), g_{F}$ is hence expected to scale as $\xi_{l o c}^{3-\eta}$ and $T_{0}$ as $\left(1-n_{B} / n_{c}\right)^{\nu \eta}$. Taking $\nu \sim 1, \eta \sim 3$, and $n_{c} \sim 4.5$ $\times 10^{20} \mathrm{~cm}^{-3}$ (see below), $T_{0}$ is expected to be rescaled by a factor of 10 for $n_{B}=2.4 \times 10^{20} \mathrm{~cm}^{-3}$ and even by a factor of 1000 for $n_{B}=4 \times 10^{20} \mathrm{~cm}^{-3}$, in reasonable agreement with our experimental values.

As discussed by several groups, ${ }^{26}$ a crossover from the Mott $(m=1 / 4)$ to the ES $(m=1 / 2)$ regime should be observed at low $T$. Such a crossover has been recently reported by Tshepe et al. ${ }^{3}$ and a progressive change from $m=1 / 4$ at high temperature to $m=1 / 2$ and finally $m=1$ at low temperature has also been reported by Sato et $a l^{24}$ for $n_{B} \sim 1.8$ $\times 10^{19} \mathrm{~cm}^{-3}$. However, it is important to note that the Coulomb gap $\Delta_{C G}$ scales as ${ }^{18} 1 / \xi^{\eta}$, leading to a vanishingly small region in which the ES regime can be observed at low temperature in our two just-insulating samples. Nevertheless, as shown in Fig. 1(b), for $n_{B}=4 \times 10^{20} \mathrm{~cm}^{-3}$ the conductivity clearly deviates from the Mott regime below $10 \mathrm{~K}$. To check for a crossover to the ES regime, we have reported in the inset of Fig. 1(b) the temperature dependence of $d \ln (\sigma) / d T \propto 1 / T^{m+1}$ in a log-log scale. At high temperature $m=1 / 4$, but the slope becomes smaller at low temperature, opposite to what is expected for the ES regime. ${ }^{26}$

At the transition, $\sigma$ is expected to scale as $1 / L \propto E^{1 / \eta}$ $\propto T^{1 / \eta}$ for finite temperatures. ${ }^{25}$ As previously reported by Tshepe et al., ${ }^{3}$ such a dependence is consistent with the deviation from the VRH law observed below $10 \mathrm{~K}$ for $n_{B}=4$ $\times 10^{20} \mathrm{~cm}^{-3}$ taking $\eta \sim 3$ [Fig. 1(b), dotted line]. This lowtemperature part of the $\sigma$ vs $T$ dependence obviously requires further investigation but a $T^{1 / 3}$ dependence has also been recently observed on a very large temperature range $(0.3$ to $\sim 50 \mathrm{~K})$ in a sample very close to the critical doping grown along the $\{111\}$ direction. ${ }^{27}$ It has been suggested by McMillan $^{25}$ that $1<\eta<3$ depending on the relative importance of one-electron localization and many-body correlation and screening effects. Measurements in disordered metals ${ }^{4}$ initially suggested that $\eta \sim 2$ but scaling analysis in doped $\mathrm{Si}$ semiconductors $^{23}$ rather suggested that $\eta \gtrsim 3$, in good agreement with numerical calculations. ${ }^{28}$ We will see in Sec. VI that this large $\eta$ value has a direct consequence for the high $T_{c}$ values observed close to the MIT.

\section{CRITICAL CONCENTRATION}

We now come to the critical concentration $n_{c}$. Figure 2 displays the low-temperature resistivity $\rho=1 / \sigma($ at $T=10 \mathrm{~K})$ as a function of the boron content together with theoretical values (solid line) obtained in a generalized Drude approach. ${ }^{29}$ In this model, the static resistivity can be written as

$$
\rho=\frac{16 \hbar k_{F}^{3}}{12 \pi n_{B} E_{F}} \int_{0}^{\infty} Q^{2} \frac{\partial \alpha_{2}(Q, W) /\left.\partial W\right|_{W=0}}{\left[\epsilon+\alpha_{1}(Q, 0)\right]^{2}} d Q,
$$

where $W=\hbar \omega / 4 E_{F}, Q=q / 2 k_{F}$, and $\alpha_{1}$ and $\alpha_{2}$ are related to the dielectric function through $\epsilon_{T}(q, \omega)=\epsilon+\alpha_{1}(q, \omega)$ 
$+i \alpha_{2}(q, \omega)$ ( $\epsilon$ being the dielectric constant, $E_{F}$ the Fermi level, and $k_{F}$ the Fermi wave number). It has been assumed that scattering arises from randomly distributed Coulomb impurities, and a single valence band with an effective mass $m^{*}=0.74$ and $\epsilon=5.7$ have been considered. This approach leads to resistivity values slightly lower than the experimental ones on the metallic side of the transition as it does not include quantum interference effects (see below). However, as shown, the experimental $\rho$ values tend toward the calculated ones for $n_{B} \gg n_{c}$, and this approach leads to a critical concentration on the order of $(4-5) \times 10^{20} \mathrm{~cm}^{-3}$, in good agreement with our experimental value (on the insulating side, the experimental resistivity data are lowered by VRH channels absent from the calculations). Assuming that the critical concentration can be defined by the Mott criterion $n_{c}^{1 / 3} a^{*} \sim 0.26$, one obtains a Bohr radius $a^{*} \sim 3.5 \AA$, in good agreement with calculations based on the boron excited states. The present experimental and calculated values for $n_{c}$ are 1 order of magnitude lower than that measured on ionimplanted diamond, ${ }^{3}$ where the doping efficiency of boron atoms may be considerably reduced by a non-substitutional incorporation. In particular, interstitial boron and boronvacancy pairs ${ }^{30}$ or boron dimers ${ }^{31}$ have been shown to lead to deep gap states and do not give any free carrier to the system. Finally, note that the effective number of carriers deduced from Hall-effect measurements $n_{\text {eff }}=1 /\left(R_{H} e t\right)$ is significantly larger than the number of boron atoms deduced from SIMS measurements (see inset of Fig. 2). A similar effect has also been reported by Locher et al. ${ }^{32}$ Such a difference cannot be accounted for by the presence of a corrected Hall coefficient, ${ }^{33}$ suggesting the presence of a complicated band structure including both holes and electrons.

\section{METALLIC SIDE OF THE TRANSITION: SCALING PROPERTIES OF THE ZERO-TEMPERATURE CONDUCTIVITY}

As shown in Fig. 1(a), for $n_{B} \geqslant 4.8 \times 10^{20} \mathrm{~cm}^{-3}$, the resistivity increases only slowly for decreasing temperature. On the metallic side of the MIT, $\sigma$ is expected to vary as $\left(e^{2} / \hbar \xi\right) f\left(\xi / L_{T}\right)$, where $L_{T}$ is a thermal cut-off length. For $\xi$ $\ll L_{T}, f \sim 1+\xi / L_{T}$ with $E_{T} \propto 1 / \sqrt{T}$ and hence $\sigma \propto \sqrt{T}$. Taking also into account the influence of weak localization effects $\left(\sigma \propto T\right.$ for electron-phonon scattering $\left.{ }^{34}\right)$, one finally expects

$$
\sigma=\sigma_{0}+A T^{1 / 2}+B T
$$

in good agreement with the experimental data, taking reasonable $A\left(\sim 1-10 \Omega \mathrm{cm} / \mathrm{K}^{1 / 2}\right)$ and $B(\sim 0.1-1 \Omega \mathrm{cm} / \mathrm{K})$ values (solid lines in Fig. 3). Note the minimum in the temperature dependence of the resistivity around $T=100-150 \mathrm{~K}$ [Fig. 1(a)] corresponding to the temperature for which the inelastic mean free path becomes on the order of the elastic one.

The zero-temperature conductivity $\left(\sigma_{0}\right)$ deduced from fits to the data above $T_{c}$ using Eq. (2) is displayed in Fig. 4(a) as a function of $n_{B} / n_{c}-1$ (taking $n_{c} \sim 4.5 \times 10^{20} \mathrm{~cm}^{-3}$ ). As $\sigma$ is expected to vary as $1 / \xi$, one expects ${ }^{25}$

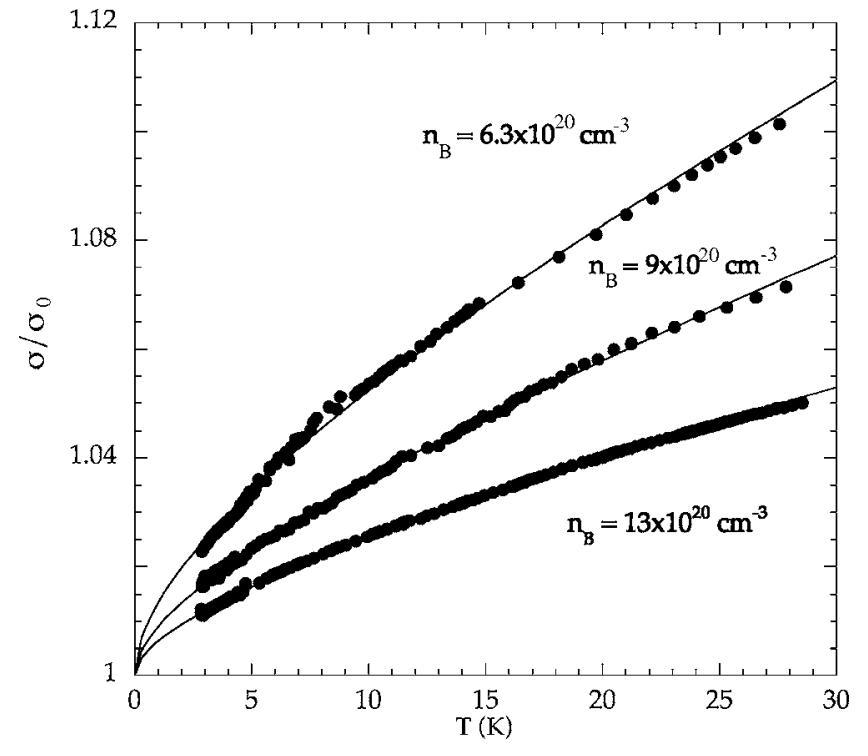

FIG. 3. Temperature dependence of the conductivity on the metallic side of the metal-insulator transition for the indicated boron concentrations. The solid lines are the fits to the data in the presence of quantum interference effects.

$$
\sigma_{0}=0.1\left(e^{2} / \hbar\right)(1 / \xi)
$$

with $a^{*} / \xi=\left(n_{B} / n_{c}-1\right)^{\nu}\left(a^{*}\right.$ being the Bohr radius $\left.\sim 3.5 \AA\right)$. As shown in Fig. 4(a) (solid line), $\sigma_{0}$ follows almost exactly the prediction of the scaling theory with $\nu \sim 1$ (without any adjustable numerical factor). In contrast to $\eta$, a unique $\nu$ value on the order of 1 has been obtained numerically in all systems whatever the relative importance of one-electron and many-body effects. This value has been confirmed in disordered metals ${ }^{4}$ as well as in many compensated [e.g., Ga:As, $\mathrm{Si}(\mathrm{P}, \mathrm{B})]$ or some uncompensated (e.g., Ge:Sb) doped semiconductors (see, for instance, Refs. 5 and 6). The $\nu=1.7$ value previously obtained by Tshepe et al. ${ }^{3}$ thus remains unexplained. However, it is important to note that $\nu \neq 1$ values have previously been reported in uncompensated $n$-type silicon based semiconductors. ${ }^{5,6,35}$ Note that the present work has been performed in the 3D limit [i.e., for film thickness $(t)$ much larger than the superconducting coherence length $\left(\xi_{0}\right)$ ], but boron-doped diamond is also a very good candidate for the study of the superconducting to insulator transition in ultrathin films. Indeed, it has been suggested that a quantum phase transition might be driven by phase fluctuations in the 2D limit $\left(\xi_{0}<t\right)$, leading to the "localization" of the Cooper pairs (so-called "dirty boson" model, for a review see Ref. 36). Even though a superconducting to insulator transition has been induced in quasi-2D ultrathin films of amorphous metals and oxides either by changing the film thickness ${ }^{37}$ or by increasing the magnetic field ${ }^{38}$ (i.e., increasing the effective disorder), the lack of a universal limiting resistance still raises questions on the nature of this transition. The main experimental limitation arises from the control of the structure and homogeneity of the films and the preparation of high quality ultrathin diamond films would thus be of fundamental interest in this topic. 


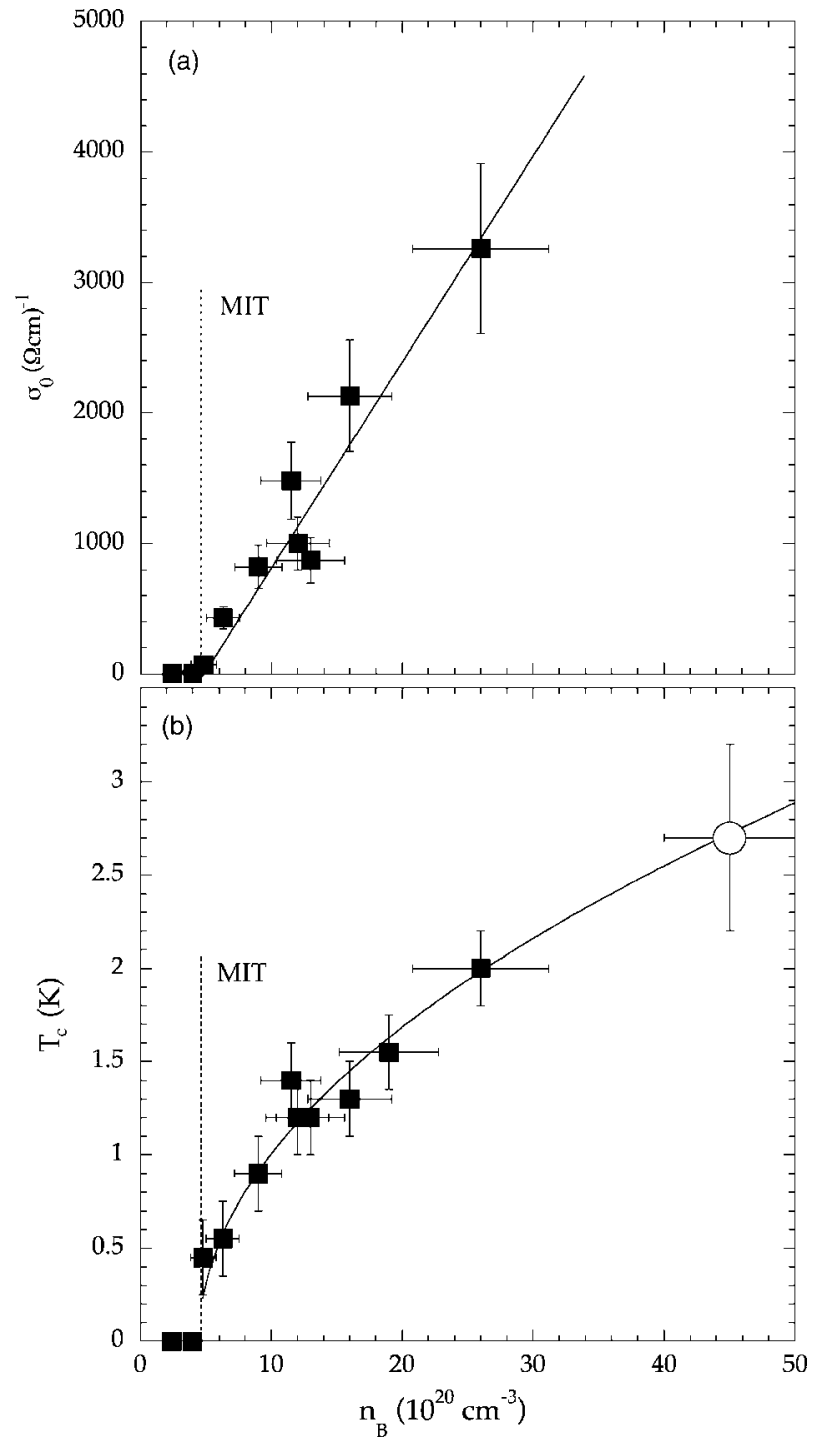

FIG. 4. (a) Conductivity extrapolated to zero temperature as a function of the boron content deduced from SIMS measurements $\left(n_{B}\right)$ in boron-doped diamond films. The solid line corresponds to the prediction of the scaling theory of the MIT taking $\nu \sim 1$ (see text for details). (b) Critical temperature as a function of the boron content deduced from SIMS measurements $\left(n_{B}\right)$ in boron-doped diamond films. The open circle has been taken from Ekimov et al. (Ref. 1). The solid line corresponds to $T_{c} \propto\left(n_{B} / n_{c}-1\right)^{0.5}$.

\section{SUPERCONDUCTIVITY}

The influence of the proximity of MIT on the superconducting properties is a long-standing puzzle which has been widely studied in disordered metals. ${ }^{39}$ It has been shown that many disordered superconductors present a dramatic enhancement of their critical temperature in the vicinity of the MIT. Soulen et al. ${ }^{4}$ suggested that this enhancement could be accounted for by the reduction of screening (of the interaction potential) and proposed to replace the Thomas-Fermi wave vector $k_{T F}$ by $k_{e f f} \propto\left(n_{B} / n_{c}-1\right)^{2 \nu}$ in the expression of the electron-phonon coupling potential $V=V_{0} /\left(\left(k_{T F} / q_{c}\right)^{2}\right.$ $+1)\left(q_{c}\right.$ being a cut-off frequency on the order of 3 times the inverse of the lattice parameter). $T_{c}$ thus first increases as the
MIT is approached following the increase of $V$ toward its unscreened $V_{0}$ value and finally drops toward zero at the MIT due to the decrease of the density of states $\left[g_{F}\right.$ is expected to scale as $\left.{ }^{25}\left(n_{B} / n_{c}-1\right)^{\nu(3-\eta)}\right]$. In disordered metal systems, $\eta=2$ (and $\nu \sim 1$ ) and the linear drop of $g_{F}$ leads to vanishingly small $T_{c}$ values close to the MIT. Note that Soulen et al. ${ }^{4}$ assumed that the Coulomb pseudopotential $\mu^{*}$ remains on the order of 0.15 . However, $\mu^{*}$ is expected to vanish at the transition and we rather assumed here that both $\lambda$ and $\mu^{*}$ are rescaled by the proximity of the MIT. Due to retardation effects, the Coulomb potential $\mu=g_{F} U / 2[U$ being the (screened) Coulomb interaction] is renormalized to

$$
\mu^{*}=\frac{\mu}{1+\mu \ln \left(\omega_{e l} / \omega_{p h}\right)},
$$

where $\hbar \omega_{e l}$ and $\hbar \omega_{p h}$ are typical electron and phonon energy scales. In metals, the electron energy scale is much larger than the phonon energy scale, $\hbar \omega_{e l} / \hbar \omega_{p h} \sim E_{F} / k_{B} \theta_{D} \sim 100$ (where $E_{F}$ and $\theta_{D}$ are the Fermi energy and Debye temperature). Therefore, $\mu^{*} \sim 1 / \ln \left(E_{F} / k_{B} \theta_{D}\right) \sim 0.15 \ll \mu$ and introducing the calculated $\lambda$ values $^{8-12}$ in Eq. (1) leads to $T_{c}$ values in good agreement with the experimental ones (on the order of a few K) when using this standard $\mu^{*} \sim 0.1-0.15$ value.

However, in doped diamond $E_{F} / k_{B} \theta_{D}<3$ and retardation effects are hence expected to be inefficient to reduce $\mu$. A somehow similar situation has been observed in alkali-doped $C_{60}$ in which superconductivity occurs in a narrow partly occupied $t_{1 u}$ subband and $\mu^{*}(\sim 0.3)$ remains close to the $\mu$ value $(\sim 0.4) .{ }^{40}$ Note that in that system, $\mu$ is reduced by efficient metallic screening. Even though the low number of carriers is expected to lead to only poor screening of the Coulomb interactions in diamond, $\mu$ is in this case expected to tend toward zero due to the proximity of a metal-insulator transition and one thus should have $\mu^{*} \sim \mu \rightarrow 0$. Both $\lambda$ and $\mu^{*}$ are thus unknown in the vicinity of the MIT.

From Eq. (1), $T_{c}$ has an exponential dependence and is not expected to follow any simple scaling law. However, as shown in Fig. 4(b), the $n_{B}$ dependence of $T_{c}$ is well described by a $\left(n_{B} / n_{c}-1\right)^{1 / 2}$ law (solid line). This emphasizes that $T_{c}$ remains remarkably large down to the MIT: $T_{c} \sim 0.4 \mathrm{~K}$ for $n_{B} / n_{c} \sim 1.1$. Indeed, such a $T_{c}$ is on the order of the one observed in metals but for a carrier concentration lower by a factor of 100-1000. Note that similar values have been recently reported in Tl-doped $\mathrm{PbTe}$ samples ${ }^{41}$ but these values are in this case assumed to be due to a peculiar coupling mechanism related to mixed valence fluctuations of $\mathrm{Tl}$ ions.

In order to extract the pseudopotential from the experimental data, it is then necessary to know the coupling constant $\lambda$. The theoretical values obtained from $a b$ initio calculations in a supercell approximation ${ }^{8,9}$ are displayed in Fig. 5(a) (open symbols). Similar values were obtained in virtualcrystal calculations ${ }^{10-12}$ (closed symbols). As shown, even if all calculations agree on a $\lambda$ value on the order of $0.4-0.5$ for $n_{B} \sim 10^{22} \mathrm{~cm}^{-3}$, the dispersion is quite large and supercell calculations in the experimental low doping range are still lacking due to computational limitations. The shaded areas in Fig. 5 schematically represent the ensemble of $\left\{\lambda, \mu^{*}\right\}$ 


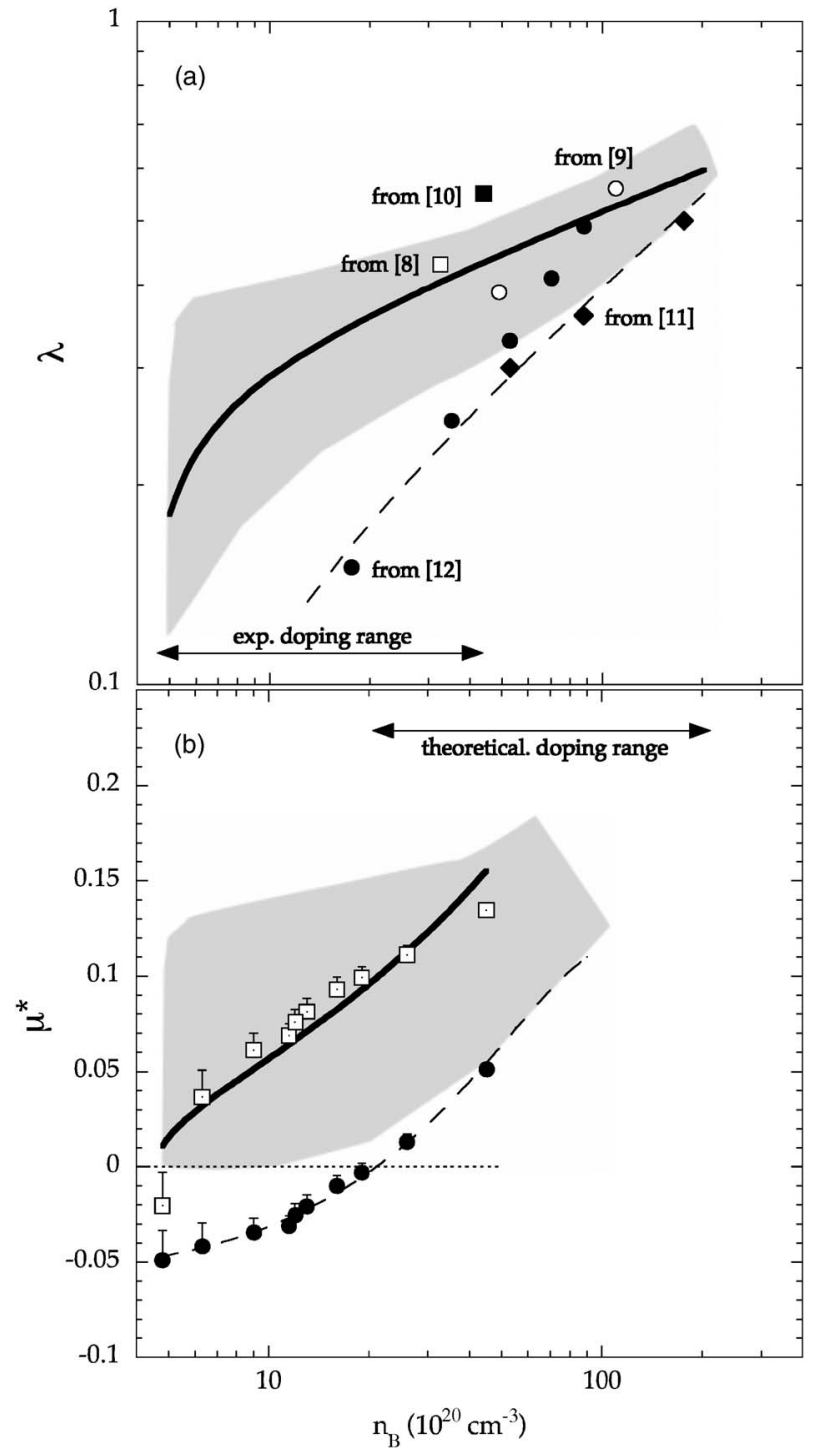

FIG. 5. (a) $\lambda$ parameter deduced from calculations in the supercell approximation (open square, from Ref. 8; open circles, from Ref. 9) and virtual-crystal approximation (closed square, from Ref. 10; closed diamonds, from Ref. 11; closed circles, from Ref. 12). The solid and dashed lines correspond to $\lambda \propto\left(n_{B} / n_{c}-1\right)^{\beta}$ laws with $\beta \sim 0.2$ and $\sim 0.5$, respectively. The corresponding $\mu^{*}$ values are displayed in (b) (open squares and closed circles, respectively) introducing the experimental $T_{c}$ values in the McMillan equation. The shaded areas correspond to $\left\{\lambda, \mu^{*}\right\}$ couples compatible with our $T_{c}$ values.

couples compatible with our $T_{c}$ values and the theoretical $\lambda$ values obtained for large doping concentrations.

Even though calculations have only been performed in the upper limit of the experimental doping range, it is tempting to extrapolate those values toward $n_{c}$ assuming that $\lambda$ will scale as $\lambda=\lambda_{a}\left(n_{B} / n_{c}-1\right)^{\beta}$. Indeed, since no maximum in the $T_{c}\left(n_{B}\right)$ curve has been observed so far, $k_{\text {eff }}$ is probably much smaller than $q_{c}$ and one expects $\lambda \propto g_{F} \propto\left(n_{B} / n_{c}-1\right)^{\nu(3-\eta)}$. Typical attempts have been reported in Fig. 5(a) for $\beta \sim 0.2$ (solid line) and $\beta \sim 0.5$ (dashed line). The latter reproduces very well the $\lambda$ values deduced from supercell calculations for large doping but, as shown, such a rapid decrease of $\lambda$ is not compatible with our experimental $T_{c}$ values as it would lead to unrealistic negative $\mu^{*}$ values [see closed circles and dashed line in Fig. 5(b)]. Note that those calculations do not take into account the possible coupling of electrons with local boron-related vibration modes, thus possibly underestimating $\lambda$. The importance of those low-energy modes has been recently pointed out by Ortolani et al. ${ }^{42}$ from optical measurements and our $T_{c}$ values confirm that those modes can play a significant role leading to large coupling constants.

As the Coulomb interaction potential is expected to be proportional to $E_{F}$ and $g_{F} \propto p / E_{F}$ ( $p$ being the carrier density), one obtains that $\mu$ (and hence $\mu^{*}$ ) should scale as $p$. Assuming that $g(E) \propto\left(1-E / E_{v}\right)^{\alpha}$ (where $E_{v}$ is the top of the valence band), one obtains $\mu^{*} \propto p=\int_{E_{F}}^{E_{v}} g(E) d E \propto(1$ $\left.-E_{F} / E_{v}\right)^{\alpha+1} \propto\left(n_{B} / n_{c}\right)^{\zeta}$ with $\zeta=\beta(\alpha+1) / \alpha$. The solid lines in Fig. 5 correspond to $\beta \sim 0.2$ and $\zeta \sim 0.5$. Note that this $\zeta$ value is in very good agreement with scaling exponents previously obtained in doped semiconductors $(0.3 \leqslant \zeta \leqslant 0.7$, see Ref. 23 and references therein) and would correspond to $\alpha$ $\sim 0.7$, e.g., close to its $\alpha=0.5$ classical value.

The main point here is that $\lambda$ has to remain relatively large down to the transition in order to reproduce the high $T_{c}$ values without introducing unrealistic negative $\mu^{*}$ values. This means that $\beta$ has to be very low (typically $\leqslant 0.3$ ). As $\beta=\nu(3-\eta)$ and $\nu \sim 1$, one obtains that $\eta \geqslant 2.7$ in diamond, in good agreement with transport data for for $n_{B} \sim n_{c}\left(\sigma \propto T^{1 / \eta}\right.$, see discussion in Sec. III). This situation is then particularly interesting as it leads to a density of states decaying only very slowly down to the close vicinity of the MIT and to $\lambda$ values remaining on the order of 0.3-0.5 down to $n_{B} / n_{c}$ $\leqslant 1.1$. Finally, note that scaling analysis in doped $\mathrm{Si}$ semiconductors even suggested that $\eta \geqslant 3$ in this compound, ${ }^{23}$ which would give rise to an enhanced density of states close to the transition.

\section{CONCLUSION}

To conclude, we have shown that boron-doped diamond presents a metal-insulator transition for a boron concentration $\left(n_{B}\right)$ on the order of $n_{c}=4.5 \times 10^{20} \mathrm{~cm}^{-3}$. The temperature dependence of the conductivity and the effective number of carriers deduced from Hall effect can be very well described by variable range hopping for $n_{B}<n_{c}$ and the characteristic hopping temperature $T_{0}$ tends toward zero for $n_{B}$ $\rightarrow n_{c}$. On the metallic side of the transition, the zerotemperature conductivity $\sigma_{0} \propto\left(n_{B} / n_{c}-1\right)^{\nu}$ with $\nu \sim 1$, in good agreement with numerical calculations. ${ }^{25}$

The critical temperature in diamond roughly behaves as $\left(n_{B} / n_{c}-1\right)^{1 / 2}$, emphasizing the fact that $T_{c}$ remains remarkably large down to the close vicinity of the MIT. This phenomenological law can be accounted for by a slow decrease of the coupling constant and corresponding collapse of the Coulomb pseudopotential. This slow decrease of $\lambda$ is consistent with a critical exponent $\eta$ being on the order of 3, indicating that the density of states remains large down to the transition. Direct measurements of $g_{F}$ as a function of $n_{B}$ will now be of fundamental interest to confirm this point. 
${ }^{1}$ E. A. Ekimov, V. A. Sidorov, E. D. Bauer, N. N. Melonik, N. J. Curro, J. D. Thompson, and S. M. Stishov, Nature (London) 428, 542 (2004).

${ }^{2}$ See, for instance, D. Belitz and T. R. Kirkpatrick, Rev. Mod. Phys. 66, 261 (1994).

${ }^{3}$ T. Tshepe, C. Kasl, J. F. Prins, and M. J. R. Hoch, Phys. Rev. B 70, 245107 (2004).

${ }^{4}$ G. Hertel, D. J. Bishop, E. G. Spencer, J. M. Rowell, and R. C. Dynes, Phys. Rev. Lett. 50, 743 (1983); R. J. Soulen, M. S. Osofsky, and L. D. Cooley, Phys. Rev. B 68, 094505 (2003).

${ }^{5}$ P. Dai, Y. Zhang, and M. P. Sarachik, Phys. Rev. Lett. 66, 1914 (1991).

${ }^{6}$ M. Watanabe, Y. Ootuka, K. M. Itoh, and E. E. Haller, Phys. Rev. B 58, 9851 (1998).

${ }^{7}$ N. F. Mott, J. Non-Cryst. Solids 1, 1 (1968).

${ }^{8}$ X. Blase, C. Adessi, and D. Connetable, Phys. Rev. Lett. 93, 237004 (2004).

${ }^{9}$ H. J. Xiang, Z. Li, J. Yang, J. G. Hou, and Q. Zhu, Phys. Rev. B 70, 212504 (2004).

${ }^{10}$ K. W. Lee and W. E. Pickett, Phys. Rev. Lett. 93, 237003 (2004).

${ }^{11}$ L. Boeri, J. Kortus, and O. K. Andersen, Phys. Rev. Lett. 93, 237002 (2004).

${ }^{12}$ Y. Ma, J. S. Tse, T. Cui, D. D. Klug, L. Zhang, Yu Xie, Y. Niu, and G. Zou, Phys. Rev. B 72, 014306 (2005).

${ }^{13}$ J. Kortus, I. I. Mazin, K. D. Belashchenko, V. P. Antropov, and L. L. Boyer, Phys. Rev. Lett. 86, 4656 (2001); J. M. An and W. E. Pickett, ibid. 86, 4366 (2001); A. Y. Liu, I. I. Mazin, and J. Kortus, ibid. 87, 087005 (2001).

${ }^{14}$ W. L. McMillan, Phys. Rev. 167, 331 (1968).

${ }^{15}$ E. Bustarret, J. Kacmarcik, C. Marcenat, E. Gheeraert, C. Cytermann, J. Marcus, and T. Klein, Phys. Rev. Lett. 93, 237005 (2004)

${ }^{16}$ J. Kacmarcik, C. Macrrenat, C. Cytermann, A. Ferreir ada Silva, L. Ortega, F. Gustafsson, J. Marcus, T. Klein, E. Gheeraert, and E. Bustarret, Phys. Status Solidi A 202, 2160 (2005).

${ }^{17}$ B. Sacepe, C. Chapelier, C. Marcenat, J. Kacmarcik, T. Klein, M. Bernard, and E. Bustarret, Phys. Rev. Lett. 96, 097006 (2006).

${ }^{18}$ A. L. Efros and B. I. Shklovskii, J. Phys. C 8, L49 (1975); 9, 2021 (1976).

${ }^{19}$ Note that the variation of the $\exp \left[-\left(T_{0} / T\right)^{m}\right]$ term becomes small close to the MIT and the temperature dependence of the prefactor $\left(\sigma_{0} \propto 1 / T^{s}\right)$ should be taken into account. The small deviation observed at high temperature for $n_{B} \leqslant 4 \times 10^{20} \mathrm{~cm}^{-3}$ can, for instance, be well corrected taking $s=1 / 2$ as predicted in the Mott regime.

${ }^{20}$ M. Gruenewald, H. Mueller, P. Thomas, and D. Wuertz, Solid State Commun. 38, 1011 (1981). For a discussion of the Hall effect in the VRH regime, see also L. Friedman and M. Pollak, Philos. Mag. B 44, 487 (1981).

${ }^{21}$ Note that $n_{B} / n_{c} \sim 0.6$ although this value of the temperature ratio has been derived in the vicinity of the metal-insulator transition [D. W. Koon and T. G. Castner, Phys. Rev. B 41, 12054 (1990)].

${ }^{22}$ V. Ambegaokar, B. I. Halperin, and J. S. Langer, Phys. Rev. B 4, 2612 (1971); M. Ortuno and M. Pollak, J. Non-Cryst. Solids
59-60, 53 (1983); A. S. Skal and B. I. Shklovskii, Sov. Phys. Solid State 16, 1190 (1974).

${ }^{23}$ T. G. Castner, in Hopping Transport in Solids, edited by M. Pollak and B. I. Schlovskii (Elsevier, Amsterdam, 1991); Phys. Rev. B 55, 4003 (1997).

${ }^{24}$ T. Sato, K. Ohashi, H. Sugai, T. Sumi, K. Haruna, H. Maeta, N. Matsumoto, and H. Otsuka, Phys. Rev. B 61, 12970 (2000).

${ }^{25}$ W. L. McMillan, Phys. Rev. B 24, 2739 (1981), and references therein.

${ }^{26}$ A. Aharony, Y. Zhang, and M. P. Sarachik, Phys. Rev. Lett. 68, 3900 (1992); Y. Meir, ibid. 77, 5265 (1996); R. Rosenbaum, Nguyen V Lien, M. R. Graham, and M. Witcomb, J. Phys.: Condens. Matter 9, 6247 (1997).

${ }^{27} \mathrm{P}$. Achatz (private communication).

${ }^{28}$ T. Ohtsuki and T. Kawarabayash, J. Phys. Soc. Jpn. 66, 314 (1997).

${ }^{29}$ A. Ferreira da Silva, Bo E. Sernelius, J. P. de Souza, H. Boudinov, H. Zheng, and M. P. Sarachik, Phys. Rev. B 60, 15824 (1999).

${ }^{30}$ J. P. Goss, P. R. Briddon, S. J. Sque, and R. Jones, Phys. Rev. B 69, 165215 (2004).

${ }^{31}$ E. Bourgeois, E. Bustarret, P. Achatz, F. Omnès, and X. Blase, Phys. Rev. B 74, 094509 (2006).

${ }^{32}$ R. Locher, J. Wagner, F. Fuchs, M. Maier, P. Gonon, and P. Koidl, Diamond Relat. Mater. 4, 678 (1995).

${ }^{33}$ This correction is expected to be smaller than 2 [F. Szmulowicz, Phys. Rev. B 34, 4031 (1986)].

${ }^{34}$ See G. Bergmann, Phys. Rep. 107, 1 (1984), and references therein.

${ }^{35}$ H. Stupp, M. Hornung, M. Lakner, O. Madel, and H. v. Lohneysen, Phys. Rev. Lett. 71, 2634 (1993).

${ }^{36}$ A. M. Goldman and N. Markovic, Phys. Today 51 (11), 39 (1998)

${ }^{37}$ See, for instance, Y. Liu, K. A. McGreer, B. Nease, D. B. Haviland, G. Martinez, J. W. Halley, and A. M. Goldman, Phys. Rev. Lett. 67, 2068 (1991); Y. Liu, D. B. Haviland, B. Nease, and A. M. Goldman, Phys. Rev. B 47, 5931 (1993).

${ }^{38}$ See, for instance, A. F. Hebard and M. A. Paalanen, Phys. Rev. Lett. 65, 927 (1990); G. Sambandamurthy, L. W. Engel, A. Johansson, E. Peled, and D. Shahar, ibid. 94, 017003 (2005).

${ }^{39}$ M. S. Osofsky, R. J. Soulen, Jr., J. H. Claassen, G. Trotter, H. Kim, and J. S. Horwitz, Phys. Rev. Lett. 87, 197004 (2001); Phys. Rev. B 66, 020502(R) (2002).

${ }^{40}$ O. Gunnarsson and G. Zwicknagl, Phys. Rev. Lett. 69, 957 (1992); E. Koch, O. Gunnarsson, and R. M. Martin, ibid. 83, 620 (1999).

${ }^{41}$ Y. Matsushita, H. Bluhm, T. H. Geballe, and I. R. Fisher, Phys. Rev. Lett. 94, 157002 (2005); Y. Matsushita, P. A. Wianecki, T. H. Geballe, and I. R. Fisher, arXiv:cond-mat/0605717 (unpublished).

${ }^{42}$ M. Ortolani, S. Lupi, L. Baldassarre, U. Schade, P. Calvani, Y. Takano, M. Nagao, T. Takenouchi, and H. Kawarada, Phys. Rev. Lett. 97, 097002 (2006). 